Pacific Journal of Mathematics

ASPECT OF Atroustc CoNtr 


\section{SOME ASPECTS OF AUTOMATIC CONTINUITY}

JAMES D. STEIN, JR.

This paper deals with problems concerning the continuity of various linear maps between Banach spaces. In the first section, it is shown that if an algebra with automatic continuity properties is mapped reasonably into another algebra, that other algebra has automatic continuity properties. In the second section, properties of ideals crucial to automatic continuity questions are investigated. The third section deals with linear maps of regular commutative semi-simple Banach algebras. The last section concerns automatic continuity questions when restrictions are placed on the range of the maps.

Let $X, Y$ be topological vector spaces, $T$ a linear map from $X$ into $Y$. Questions of automatic continuity can, in general, be stated as follows: what structural restrictions on $X$ and $Y$, and what hypotheses on $T$, are sufficient to ensure the continuity of $T$ ? Unless $X$ is finitedimensional, it is, of course, necessary to place some hypotheses on the nature of $T$. In general, automatic continuity theorems can be classified into two major categories: Those in which the primary restrictions are placed on the domain space $X$, and those in which the primary restrictions are placed on the range space $Y$. In this paper we shall investigate both types of theorems. All maps will be assumed to be linear.

1. Separable maps and module actions. The following concept was introduced in [9]; we present here a slight but useful generalization.

Definition 1.1. Let $A, B, X, Y$ be normed spaces, $q: A \times B \rightarrow X$ a continuous bilinear form. Let $T: X \rightarrow Y$ be linear, and let $R^{+}$denote the positive reals. We say $T$ is separable (with respect to $q$ ) if there exist functions $f: A \rightarrow R^{+}, g: B \rightarrow R^{+}$such that $\|T(q(a, b))\| \leqq f(a) g(b)$ for all $a \in A, b \in B$.

The proof of the following basic lemma is a trivial modification of ([9], Lemma 1.1), and so will be omitted.

Lemma 1.1. Let $A, B$ be Banach spaces, $X, Y$ normed spaces, $q: A \times B \rightarrow X$ a continuous bilinear form, and $T: X \rightarrow Y$ a linear map separable with respect to $q$. Let $\left\{a_{n}\right\} \subseteq A,\left\{b_{k}\right\} \subseteq B$ such that $q\left(a_{n}, b_{k}\right)=0$ if $n \neq k$. Then

$$
\sup _{n}\left\|T\left(q\left(a_{n}, b_{n}\right)\right)\right\| /\left\|a_{n}\right\|\left\|b_{n}\right\|<\infty .
$$


There are two important examples of interesting bilinear forms $q$ and related separable maps. The first example of an interesting bilinear form is algebra multiplication, i.e., $A=B=X$ and $q(a, b)=a b$. The second is module multiplication. Let $X$ be a Banach $A$-module (a Banach space with a continuous bilinear map $(a, x) \rightarrow a x$ which satisfies the usual associativity relationship).

Lemma 1.2. Let $X, Y$ be Banach spaces, $Z$ a normed linear space. Let $\pi: X \rightarrow Y$ be continuous and onto, and let $T: Y \rightarrow Z$ be linear. Then $T$ is continuous iff $T \circ \pi$ is continuous.

Proof. Suppose that $T \circ \pi$ is continuous, and let $y_{n} \rightarrow 0$ in $Y$. Then there is a sequence $\left\{x_{n}\right\}$ in $X$ such that $x_{n} \rightarrow 0$ and $\pi\left(x_{n}\right)=y_{n}$. So $T y_{n} \rightarrow 0$.

We are thus led to the following diagram:

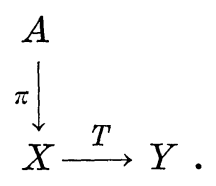

It is clearly unreasonable for us to attempt to obtain nontrivial results by making assumptions about $T \circ \pi$, so we shall make assumptions concerning the structure of $A$ and the nature of $T$ and $\pi$.

The time has now come to introduce some ideals that are extremely useful in the study of automatic continuity questions.

Definition 1.2. Let $A$ be a Banach algebra, $Y$ a normed space, $T: A \rightarrow Y$. Let $J_{L}=\{a \in A \mid b \rightarrow T(b a)$ is continuous $\}, J_{R}=\{a \in A \mid b \rightarrow$ $T(a b)$ is continuous $\}$ and $J=\{a \in A \mid(b, c) \rightarrow T(b a c)$ is continuous $\}$.

$J_{L}$ is a left ideal, $J_{R}$ is a right ideal, and $J$ is a two-sided ideal. If there are several maps under consideration, we shall refer to $J_{L}(T)$, etc. If $A$ is known to be commutative, we shall let $J$ denote $J_{L}=J_{R}$; hopefully, no confusion will result.

Definition 1.3. A Banach algebra $A$ will be called an automatic algebra if every linear map $T: A \rightarrow Y$ which is separable with respect to algebra multiplication is continuous. $A$ will be called a semiautomatic algebra if every linear map $T: A \rightarrow Y$ which is separable with respect to algebra multiplication and such that $J$ is a closed twosided ideal is continuous.

Examples of automatic algebras are the continuous Von Neumann algebras (see [9], § 2); and a modification of Ringrose's proof that every module-valued derivation of a $C^{*}$-algebra is continuous ([10]) will show 
that $C^{*}$-algebras are semi-automatic.

Proposition 1.1. Let $A$ be an automatic algebra, $X$ a Banach space, $Y$ a normed space. Let $T: X \rightarrow Y$ be linear, $\pi: A \rightarrow X$ continuous and onto such that $T \circ \pi$ is separable. Then $T$ is continuous.

\section{Proof. Immediate from Lemma 1.2.}

There is, of course, an obvious analogue of Proposition 1.1 for semiautomatic algebras; in addition to all the above hypotheses we must require that $J(T \circ \pi)$ be closed. This leads to two questions. First, suppose that $T$ is separable; when is the lift $T \circ \pi$ separable? Second, suppose that the ideal $J(T)$ is closed; when is the ideal $J(T \circ \pi)$ also closed?

We repeat here a definition from ([9]).

Definition 1.4. A Banach algebra $A$ is called a Johnson algebra if it contains an infinite sequence of orthogonal idempotents, and there are no proper two-sided ideals which contain an infinite sequence of orthogonal idempotents.

Our next goal is the following proposition, the utility of which derives from the fact that $\pi$ is not required to be surjective, nor even to have closed range.

Proposition 1.2. Let $A$ be a Johnson algebra, $X$ a Banach algebra, and suppose $\exists$ continuous maps $L, R: A \rightarrow X$ such that a continuous map $\pi: A \rightarrow X$ satisfies $\pi(a b)=L(a) R(b)$ for $a, b \in A$. Let $T: X \rightarrow Y$ be separable with respect to algebra multiplication. Then, for any $a \in A$, we have

$$
\sup \{\|T(\pi(b a c) x)\| \mid b, c \in A, x \in X,\|b\| \leqq 1,\|c\| \leqq 1,\|x\| \leqq 1\}<\infty .
$$

Proof. For convenience, let $S=\{(b, c, x) \in A \times A \times X \mid\|b\| \leqq 1$, $\|c\| \leqq 1,\|x\| \leqq 1\}$, and let $I=\{a \in A \mid(b, c, x) \rightarrow T(\pi(b a c) x)$ is continuous on $A \times A \times X\}$. If $a \in I$ and $b^{\prime}, c^{\prime} \in A$, then $(b, c, x) \rightarrow\left(b b^{\prime}, c, x\right) \rightarrow$ $T\left(\pi\left(b\left(b^{\prime} a\right) c\right) x\right)$ and $(b, c, x) \rightarrow\left(b, c^{\prime} c, x\right) \rightarrow T\left(\pi\left(b\left(a c^{\prime}\right) c\right) x\right)$ are continuous, so $I$ is a two-sided ideal. Define $q: A \times X \rightarrow X$ by $q(a, x)=L(a) x ; q$ is clearly a continuous bilinear form. Note that, if $e$ is an idempotent, $\pi(b e c) x=\pi((b e)(e c)) x=L(b e) R(e c) x=q(b e, R(e c) x)$. Note also that if $a, b \in A$ and $a b=0,0=\pi(a b)=L(a) R(b)$.

Let $\left\{e_{n}\right\}$ be an orthogonal sequence of idempotents in $A$; we are done if we can show $\exists N$ such that $n \geqq N \Rightarrow e_{n} \in I$. Suppose not, and by re-numbering if necessary assume that no $e_{n} \in I$. Recalling that $R$ is continuous, we can therefore choose, for each integer $n$, a triple 
$\left(b_{n}, c_{n}, x_{n}\right) \in S$ such that $\left\|T\left(\pi\left(b_{n} e_{n} c_{n}\right) x_{n}\right)\right\|>n\left\|e_{n}\right\|^{2}\|R\|$. Consider the sequences $\left\{b_{n} e_{n}\right\} \subseteq A,\left\{R\left(e_{n} e_{n}\right) x_{n}\right\} \subseteq X$. Then $n \neq m \Rightarrow q\left(b_{n} e_{n}\right.$, $\left.R\left(e_{m} c_{m}\right) x_{m}\right)=L\left(b_{n} e_{n}\right) R\left(e_{m} c_{m}\right) x_{m}=0$, and so by Lemma 1.1, $\sup _{n} \| T\left(q\left(b_{n} e_{n}\right.\right.$, $\left.\left.R\left(e_{n} c_{n}\right) x_{n}\right)\right)\|/\| b_{n} e_{n}\|\| R\left(e_{n} c_{n}\right) x_{n} \|<\infty$. But $n\left\|b_{n} e_{n}\right\|\left\|R\left(e_{n} c_{n}\right) x_{n}\right\| \leqq$ $n\left\|e_{n}\right\|^{2}\|R\|<\left\|T\left(\pi\left(b_{n} e_{n} c_{n}\right) x_{n}\right)\right\|=\left\|T\left(q\left(b_{n} e_{n}, R\left(e_{n} c_{n}\right) x_{n}\right)\right)\right\|$, a contradiction which, along with the structure of a Johnson algebra, establishes the desired result.

One obvious corollary occurs when the algebra $X$ has an identity, and the range of the map $\pi$ contains this identity. Then every separable map of $X$ is continuous. A consequence of this is the following result.

Corollary 1.2.1. Let $A$ be a Johnson algebra with identity, $X$ a Banach algebra with identity, $\pi: A \rightarrow X$ a homomorphism (which is automatically continuous, by [6], Theorem 3.3) such that the image of the identity of $A$ is the identity of $X$. Then $X$ has a unique Banach algebra topology.

Proof. If $(X,\|\cdot\|)$ and $(X,|\cdot|)$ are two normed algebra topologies for $X$, the identity $i:(X, \| \cdot||) \rightarrow(X,|\cdot|)$ is a homeomorphism by Proposition 1.2 .

A precursor of Proposition 1.2 can be found in [11], where it is observed that tensoring Johnson algebras with Banach algebras with identity yields a Johnson algebra.

A crucial ingredient of the proof of Proposition 1.2 is the ability to take the element $\pi(a b) x$ and "associate" $b$ with $x$ by means of the equality $\pi(a b) x=L(a)[R(b) x]$. Another instance in which this "association" can be performed is when $X$ is an $A$-module. The following proposition can be proved in a manner similar to Proposition 1.2.

Proposition 1.3. Let $A$ be a Johnson algebra, $X$ a Banach $A$-module. Let $q: A \times X \rightarrow X$ be the continuous bilinear form defined by module multiplication, and assume that $T: X \rightarrow Y$ is separable with respect to this bilinear form. Then for each $a \in A$,

$$
\sup \{\|T(b a c x)\| \mid(b, c, x) \in A \times A \times X,\|b\| \leqq 1,\|c\| \leqq 1,\|x\| \leqq 1\}<\infty .
$$

We digress for a moment to mention an attraction of the $A$-module concept. Let $K$ denote the usual Cantor set, $C(K)$ the algebra of all continuous complex functions on $K$. It is unknown as to whether or not there are any discontinuous homomorphisms of $C(K)$ (or any nontrivial $C(\Omega)$, for that matter) into a normed algebra. Let $B(C(K))$ denote the algebra of all bounded linear operators on the Banach space 
$C(K)$; since $C(K)$ is a Banach space with a continued bisection (see [6] for the relevant definitions and theorems), $B(C(K))$ is a Johnson algebra. Moreover, $C(K)$ has a natural $B(C(K))$-module structure, namely if $f \in C(K), T \in B(C(K))$, multiplication is defined by $T f$. The problem here is that separability of a map of $C(K)$ with respect to algebra multiplication into a normed linear space does not a priori induce separability of that same map with respect to module multiplication. In fact, about the only time one can conclude that separability with respect to algebra multiplication implies separability with respect to module multiplication is when both $A$ and $X$ are commutative and $X$ has an identity $e$. In this case, if $a \in A$ and $x \in X$, we have $T(a x)=$ $T((a e)(e x))$, and since $a e \in X$ we see that the above statement holds.

Consider now the following question: let $X, Y$ be Banach spaces, and let $T, \hat{T}: X \rightarrow Y$ be linear maps. Let $A$ be a sub-algebra of $B(X)$, and suppose that for each $S \in A$, we can find an $\hat{S} \in B(Y)$ such that for each $x \in X, T(S x)=\widehat{S}(\widehat{T} x)$. What can be said about the continuity of $T$ ?

In this instance we make use of the fact that $X$ is a $B(X)$-module in the obvious fashion, $q: B(X) \times X \rightarrow X$ is defined by $q(S, x)=S x$. Since $T(q(S, x))=T(S x)=\widehat{S}(\widehat{T} x)$, we have $\|T(q(S, x))\| \leqq\|\widehat{S}\|\|\widehat{T} x\| \leqq$ $\max (\|\hat{S}\|, 1) \max (\|\hat{T} x\|, 1)$, and so $T$ is separable with respect to $q$. Utilizing this relationship, one can obtain generalizations of the results of Griffin ([4]), who considered this problem when $A$ was an algebra of operators on a Hilbert space $H$, and $\hat{S}=S, Y=X=H$.

Note that the results of Propositions 1.2 and 1.3 require that $A$ be stronger than just an automatic algebra (the infinite-dimensionality of Johnson algebras is critical), for if $A=\mathscr{C}$, the complex numbers, and $X$ is any complex Banach algebra with an identity $e$, the map $\pi: A \rightarrow X$ defined by $\pi(s)=s e$ satisfies the conditions of Proposition 1.2, but it is clearly ridiculous to expect every separable map of $X$ to be continuous.

The useful fact about Johnson algebras is that virtually any ideal that is related somehow to continuity questions is the entire algebra. The algebraic structure is powerful enough to overcome most obstacles. However, Lemma 1.1 guarantees that, in any algebra rich in orthogonal elements, any continuity related ideal will tend to be large.

Now let $A$ be a $C^{*}$-algebra. Although the algebra structure of $A$ is no longer as powerful as that of a Johnson algebra, it is quite rich in orthogonal elements. We shall show that, if the map $T$ is sufficiently restricted, a two-sided continuity related ideal (defined in much the same manner as the ideal $I$ in Proposition 1.2) will turn out to be closed and cofinite. This idea, of course, derives from Ringrose's argument on module derivations of $C^{*}$-algebras, in which he shows that, if $T$ is such a derivation, the ideal $J_{L}$ is two-sided, closed, and cofinite, 
and in addition $T \mid J_{L}$ is continuous. These facts are sufficient to ensure the continuity of $T$. For our problem, however, this will not in general suffice, since the ideal we will consider is contained in $A$ rather than $X$.

Although the work done now is intended to apply to $C^{*}$-algebras, later in this paper we shall show that it is applicable to other algebras as well, one of which is the algebra of all absolutely convergent Fourier series.

Proposition 1.4. Let $A$ be a $C^{*}$-algebra, $X$ a Banach algebra, $\pi: A \rightarrow X$ a homomorphism. Let $Y$ be a normed space, $T: X \rightarrow Y$ separable with respect to algebra multiplication. Let $I=\{a \in A \mid(x, y) \rightarrow$ $T(x \pi(a) y)$ is continuous $\}$. Then $I$ is a two-sided ideal in $A$ with cofinite closure.

Proof. If $a \in I, b \in A$, then $(x, y) \rightarrow(x \pi(b), y) \rightarrow T(x \pi(b) \pi(a) y)=$ $T(x \pi(b a) y)$ is continuous, so $I$ is a left ideal; similarly it is also a right ideal.

We now assert that, if $\left\{a_{n}\right\},\left\{b_{m}\right\}$ are sequences from $A$ such that $a_{n} b_{m}=0$ if $n \neq m$, then $\exists N$ such that $n \geqq N \Rightarrow a_{n} b_{n} \in I$. The proof is sufficiently similar to a portion of Proposition 1.2 to eliminate the necessity of establishing the assertion. The fact that the closure of $I$ is cofinite now follows from Lemmas 2.3, 2.4, and 2.5 of [7].

First of all, note that $\pi$ is not required to be continuous (although it may be possible to establish that it is, depending upon the structure of $A$ and $X$ ). There are some attractive corollaries available.

Corollary 1.4.1. If $\pi$ is continuous and $J(T)$ is closed, then $I$ is closed.

Proof. Note that $I=\pi^{-1}(J(T))$, and the assertion follows.

A simple example shows that, even in the circumstances specified by Corollary 1.4.1, $A$ need not be equal to $I$. Let $A=\mathscr{C}$, the complex numbers, and let $X$ be a Banach algebra with an identity $e$ and a discontinuous module derivation $T: X \rightarrow Y . J(T)$ is closed, and define $\pi: A \rightarrow X$ by $\pi(s)=s e$. Then either $I=(0)$ or $I=A$. But if $I=A$, then $T$ would be continuous.

However, there are examples of $C^{*}$-algebras whose structure is sufficiently strong, in addition to the Von Neumann algebras with no finite Type I part. 
proper closed cofinite ideals, and suppose that the image under $\pi$ of the identity of $A$ is the identity of $X$. Then $T$ is continuous.

Proof. By the hypotheses, $\bar{I}=A$, so $I$ contains elements of $A$ where distance from the identity is less than 1 . These elements are invertible, so $I=A$, and the result follows.

Results analogous to these can of course be obtained for the case when $\mathrm{X}$ is an $A$-module, as long as the given map $T$ is separable with respect to the module multiplication. These results will parallel Proposition 1.4 and its corollaries in the same way that Proposition 1.3 parallels Proposition 1.2.

The results of this section can roughly be paraphrased as follows: if a reasonable algebra $A$ can be reasonably mapped into a Banach algebra $X$, then $X$ is reasonable.

2. The ideals $J_{L}, J_{R}$, and $J$. The purpose of this section is to assemble some facts concerning the ideals $J_{L}=\{x \in X \mid y \rightarrow T(y x)$ is continuous $\}, J_{R}=\{x \in X \mid y \rightarrow T(x y)$ is continuous $\}$, and $J=\{x \in X \mid(y, z) \rightarrow$ $T(y x z)$ is continuous $\}$, and some consequences.

Lemma 2.1. (a) Let $S$ be a closed subspace of $J_{R} \cap J_{L}$. Then $\exists K>0$ such that $s, t \in S \Rightarrow\|T(s t)\| \leqq K\|s\|\|t\|$.

(b) Let $S$ be a closed right ideal, $S \subseteq J_{R} \cap J_{L}$. Then $\exists K>0$ such that $s, t \in S, x \in X \Rightarrow\|T(s t x)\| \leqq K\|s\|\|t\|\|x\|$. If $S$ is a closed left ideal, the conclusion holds for $\|T(x s t)\|$.

(c) Let $S$ be a closed right ideal, $S \subseteq J_{R} \cap J_{L}$, such that $S$ contains a bounded left approximate identity. Then $\exists K>0$ such that $\|T(s x)\| \leqq K\|s\|\|x\|$ for $s \in S, x \in X$.

Proof. (a) is ([12], Proposition 2.1).

(b) Follows immediately from (a).

(c) By the Johnson-Varapolous extension of Cohen's Factorization Theorem, if $\left\{s_{n}\right\} \subseteq S$ and $s_{n} \rightarrow 0$, we can find $t \in S,\left\{u_{n}\right\} \subseteq S$ such that $u_{n} \rightarrow 0$ and $s=t u_{n}$ (see [5] for a proof; an attractive result, due to Taylor in [14], is that a slightly strengthened version of this simultaneous sequential factorization property is equivalent to the existence of a bounded approximate identity). For each $x \in X$ with $\|x\| \leqq 1$, define $U_{x}: S \rightarrow Y$ by $U_{x}(s)=T(s x)$. Fix $x,\|x\| \leqq 1$, and let $s_{n} \rightarrow 0$ in $S$. If $s_{n}=t u_{n}$ as above, then $U_{x}\left(s_{n}\right)=T\left(t\left(u_{n} x\right)\right) \rightarrow 0$ by part (a) of this lemma, so $U_{x}$ is continuous. For each $s \in S$, $\sup \left\{\left\|U_{x}(s)\right\| \mid x \in X\right.$, $\|x\| \leqq 1\}=\sup \{\|T(s x)\| \mid x \in X,\|x\| \leqq 1\}<\infty$ since $s \in J_{R}$. Since $S$ is closed, the result now follows from the Uniform Boundedness Principle. 
The following proposition suggests that one may be able to obtain continuity related results in the absence of an a priori separability condition.

Proposition 2.1. Let $A$ be a Banach algebra, $X$ and $Y$ be normed linear spaces, and let $\left\{\varphi_{\alpha} \mid \alpha \in \Lambda\right\}$ be a uniformly bounded family of linear maps from $X$ into $Y$. Let $T: A \rightarrow X$ be linear. For each $\alpha \in \Lambda$, let $S_{\alpha}$ be a closed subspace of $J_{R}\left(\varphi_{\alpha} \circ T\right)$ which satisfies the following condition: if $x_{n} \rightarrow 0$ and $\left\{x_{n}\right\} \subseteq S_{\alpha}, \exists y \in J_{R}\left(\varphi_{\alpha} \circ T\right),\left\{z_{n}\right\} \subseteq A$ such that $z_{n} \rightarrow 0$ and $x_{n}=y z_{n}$. Then $\exists \alpha_{1}, \cdots, \alpha_{n} \in \Lambda$ such that $\left\{\varphi_{\alpha} \circ T \mid \alpha \in \Lambda\right\}$ is equicontinuous on $\bigcap_{k=1}^{n} S_{\alpha_{k}}$.

Proof. Let $\alpha \in \Lambda$, let $x_{n} \rightarrow 0$ in $S_{\alpha}$, and factor $x_{n}=y z_{n}$ as above. Then $\varphi_{\alpha}\left(T x_{n}\right)=\varphi_{\alpha} \circ T\left(y z_{n}\right) \rightarrow 0$ since $y \in J_{R}\left(\varphi_{\alpha} \circ T\right)$, and so $\varphi_{\alpha} \circ T \mid S_{\alpha}$ is continuous. For each $x \in X,\left\|\varphi_{\alpha} \circ T(x)\right\| \leqq\left\|\varphi_{\alpha}\right\|\|T x\|$, and so $\left\{\varphi_{\alpha} \circ T \mid \alpha \in \Lambda\right\}$ is pointwise-bounded. The conclusion now follows from ([13], Theorem 1).

Before continuing let us note that, in Proposition 2.1, we can assume that $\varphi_{\alpha}: X \rightarrow Y_{\alpha}$ for some normed linear space $Y_{\alpha}$, as we can then form $\Sigma \oplus Y_{\alpha}=Y$, inject $Y_{\alpha}$ isometrically into $Y$, and proceed.

COROLlary 2.1.1. Assume that, as in the above comment, for each $x \in X$, we can find a normed linear space $Y_{x}$, an $\alpha(x) \in \Lambda$, and $a$ constant $\delta>0$ independent of $x$ such that $\left\|\varphi_{\alpha(x)}(x)\right\| \geqq \delta\|x\|$ : then $T \mid \bigcap_{k=1}^{n} S_{\alpha_{k}}$ is continuous (all other notation as in Proposition 2.1).

Proof. From Proposition 2.1, let $\left\|\varphi_{\alpha}(T a)\right\| \leqq K\|a\|$ for $a \in \bigcap_{k=1}^{n} S_{\alpha_{k}}$; then for each $a \in \bigcap_{k=1}^{n} S_{\alpha_{k}}, \delta\|T a\| \leqq\left\|\varphi_{\alpha\left(T_{a}\right)}(T a)\right\| \leqq K\|a\|$, and done.

Returning to the problem of homomorphisms of $C(X)$ for $X$ a compact Hausdorff space, let $T: C(X) \rightarrow B$ be a Banach algebra homomorphism. It is well-known ([1], Theorem 4.3) that $\overline{T(C(X))}=C(\Omega) \oplus R$, where $\Omega$ is also a compact Hausdorff space and $R$ is the Jacobson radical of $\overline{T(C(X))}$. Consider the natural projection $P: C(\Omega) \oplus R \rightarrow C(\Omega)$; for each $x \in C(\Omega)$ we have $P x=x$, and thus, for all $x \in C(\Omega)$ we can choose $\varphi_{\alpha(x)}=P$ and $\delta=1$ using the notation of Corollary 2.1.1. We are thus led to the problem of finding, for each $r \in R$, a normed linear space $Y_{r}$, and a linear map $\varphi_{r}: R \rightarrow Y_{r}$ such that the $\left\{\varphi_{r} \mid r \in R\right\}$ uniformly separate the images $\left\{\varphi_{r}(r)\right\}$ from 0 in the sense of Corollary 2.1.1. Finally, we shall want the ideals $J\left(\varphi_{r} \circ T\right)$ to be, in some sense, useful.

What type of maps would be useful in solving this problem? If $\varphi_{r}$ is a map such that the ideal $J\left(\varphi_{r} \circ T\right)$ is closed and the composite $\varphi_{r} \circ T$ is separable, then $\varphi_{r} \circ T$ is continuous; hence $J\left(\varphi_{r} \circ T\right)=C(X)$. 
But $P \circ T$ is also continuous; consequently $C(X)=\bigcap_{k=1}^{n} S_{\alpha_{k}}$, and we would be done. Note that point derivations would provide possible candidates for $\varphi_{r}$.

The next two propositions concern the relationship of the ideals $J\left(\varphi_{\alpha} \circ T\right)$; and the proofs are motivated by the technique of [8].

Proposition 2.2. Let $A$ be a commutative Banach algebra, $X, Y$ normed linear spaces. Let $\left\{\varphi_{n}\right\}$ be a sequence of continuous maps from $X$ into $Y$, and assume $\left\|\varphi_{n}\right\| \leqq 1$ for all $n$. Let $J_{n}=J\left(\varphi_{n} \circ T\right)$, where $T: A \rightarrow X$ is linear. Then, if $y_{n} \in J_{n},\left\|y_{n}\right\| \leqq 1$, we have

$$
\sup _{n} \sup _{\substack{|x| \leq \mid \leq 1 \\ x \in A}}\left\|\varphi_{n}\left[T\left(\left(\prod_{j=1}^{n} y_{j}\right) x\right)\right]\right\|<\infty \text {. }
$$

Proof. Suppose not. Choose $n_{1}, x_{1}$ such that

$$
\left\|x_{1}\right\|<1 / 2 \max \left(1, \prod_{j=1}^{n_{1}}\left\|y_{j}\right\|\right) \text { and }\left\|\varphi_{n_{1}}\left[T\left(\left(\prod_{j=1}^{n_{1}} y_{j}\right) x_{1}\right)\right]\right\|>2 \text {. }
$$

Having chosen $n_{1}<\cdots<n_{p}$ and $x_{1}, \cdots, x_{p}$, we choose $n_{p+1}$ and $x_{p+1}$ as follows: we wish to ensure the following conditions

(1) $\left\|\varphi_{n_{p+1}}\left(T\left(\prod_{j=1}^{n} p+1=y_{j}\right) x_{p+1}\right)\right\|>p+2+\sum_{k=1}^{p}\left\|T\left(\prod_{j=1}^{n_{k}} y_{j}\right) x_{k}\right\|$

(2) $\left\|\left(\prod_{j=1}^{n+1} y_{j}\right) x_{p+1}\right\|<1 / 2^{p+1}$

(3) For $1 \leqq k \leqq p$, let $M_{k}$ denote the bound of the continuous linear map $x \rightarrow \varphi_{n_{k}}\left(T\left(\left(\prod_{j=1}^{n_{k}} y_{j}\right) x\right)\right)$; it is continuous since $y_{n_{k}} \in J_{n_{k}}$, which is an ideal. We wish to have $\left\|\varphi_{n_{k}}\left(T\left(\left(\prod_{j=1}^{n_{k}} y_{j}\right)\left(\prod_{j=n_{k}+1}^{n_{p+1}} y_{j}\right) x_{p+1}\right)\right)\right\|<1 / 2^{p+1}$ for $1 \leqq k \leqq p$.

Property (3) can be accomplished by choosing

$$
\max _{1 \leqq k \leqq p}\left(1+M_{k}\right)\left\|\prod_{j=n_{k}+1}^{n_{p+1}} y_{j}\right\|\left\|x_{p+1}\right\|<1 / 2^{p+1} .
$$

All three of these conditions can be satisfied if, given $\varepsilon>0, M>0$, it is possible to find $n_{p+1}, x_{p+1}$ with

$$
\left\|x_{p+1}\right\|<\varepsilon,\left\|\varphi_{n_{p+1}}\left(T\left(\left(\prod_{j=1}^{n_{p+1}} y_{j}\right) x_{p+1}\right)\right)\right\|>M .
$$

To accomplish this, merely select $n_{p+1}$ such that

$$
\sup _{\substack{|x| \mid \leq 1 \\ x \in \mathbb{A}}}\left\|\varphi_{n_{p+1}}\left(T\left(\left(\prod_{j=1}^{n_{p+1}} y_{j}\right) x\right)\right)\right\|>M / \varepsilon
$$

choose $z_{p+1}$ attaining this inequality, $\left\|z_{p+1}\right\| \leqq 1$, and let $x_{p+1}=\varepsilon z_{p+1}$. Now let $x=\sum_{p=1}^{\infty}\left(\prod_{j=1}^{n} y_{j}\right) x_{p}$; by condition (2), $x \in A$. For each integer $p$, we have 


$$
\begin{aligned}
\varphi_{n_{p}}(T x)= & \varphi_{n_{p}}\left(\sum_{k=1}^{p-1} T\left(\left(\prod_{j=1}^{n_{k}} y_{j}\right) x_{k}\right)\right)+\varphi_{n_{p}}\left(T\left(\prod_{\jmath=1}^{n_{p}} y_{j}\right) x_{p}\right) \\
& +\varphi_{n_{p}}\left(T\left(\sum_{k=p+1}^{\infty}\left(\prod_{j=1}^{n_{k}} y_{j}\right) x_{k}\right)\right) .
\end{aligned}
$$

But

$$
\varphi_{n_{p}}\left(T\left(\sum_{k=p+1}^{\infty}\left(\prod_{j=1}^{n_{k}} y_{j}\right) x_{k}\right)\right)=\varphi_{n_{p}}\left(T\left(\prod_{j=1}^{n_{p}} y_{j}\right)\left(\sum_{k=p+1}^{\infty}\left(\prod_{j=n_{p}+1}^{n_{k}} y_{j}\right) x_{k}\right)\right) ;
$$

and so

$$
\begin{aligned}
\left\|\rho_{n_{p}}\left(T\left(\sum_{k=p+1}^{\infty}\left(\prod_{\jmath=1}^{n_{k}} y_{j}\right) x_{k}\right)\right)\right\| & \leqq M_{p}\left\|\sum_{k=p+1}^{\infty}\left(\prod_{j=n_{p}+1}^{n_{k}} y_{j}\right) x_{k}\right\| \\
& <\sum_{k=p+1}^{\infty} 2^{-k}\left(\frac{M_{p}}{1+M_{p}}\right)<1 ;
\end{aligned}
$$

and therefore,

$$
\begin{aligned}
\left\|\varphi_{n_{p}}(T x)\right\| & \geqq\left\|\rho_{n_{p}}\left(T\left(\left(\prod_{j=1}^{n_{p}} y_{j}\right) x_{p}\right)\right)\right\|-\sum_{k=1}^{p-1}\left\|\rho_{n_{p}}\left(T\left(\left(\prod_{j=1}^{n_{k}} y_{j}\right) x_{k}\right)\right)\right\|-1 \\
& \geqq\left\|\rho_{n_{p}}\left(T\left(\left(\prod_{j=1}^{n_{p}} y_{j}\right) x_{p}\right)\right)\right\|-\sum_{k=1}^{p-1}\left\|T\left(\left(\prod_{j=1}^{n_{k}} y_{j}\right) x_{k}\right)\right\|-1>p
\end{aligned}
$$

by condition (2), which leads to the fact that $\|T x\| \geqq\left\|\varphi_{n_{p}}\right\|\|T x\| \geqq$ $\left\|\varphi_{n_{p}}(T x)\right\|>p$, a contradiction.

The uniformity exhibited in the previous proposition by the sequence $\left\{\Pi_{j=1}^{n} y_{j} \mid n=1,2, \cdots\right\}$ is reminiscent, to some extent, of Lemma 1.1. Although the proof of the following proposition is quite similar, the conclusion tends to suggest that, for any collection of continuous $\left\{\varphi_{n}\right\}$, the ideals $J\left(\varphi_{n} \circ T\right)$ cannot be disjoint.

Proposition 2.3. Let $A$ be a commutative Banach algebra, $X, Y$ normed linear spaces. Let $T: A \rightarrow X$ be linear, and let $\left\{\varphi_{n}\right\}$ be a sequence of continuous linear maps of $X$ into $Y,\left\|\varphi_{n}\right\| \leqq 1$. Let $J_{n}=J\left(\varphi_{n} \circ T\right)$, and assume $\left\{y_{n}\right\} \subseteq A$ such that $y_{n+1} \in J_{n}$ for $n=1,2$, … Then $\exists N$ such that $\prod_{k=1}^{n} y_{k} \in J_{n}$ for $n>N$.

Proof. Suppose not, and choose a sequence $\left\{n_{p} \mid p=1,2, \cdots\right\}$ such that $n_{p}<n_{p+1}$ and $\prod_{k=1}^{n_{p}} y_{k} \notin J_{n_{p}}$. Note that, as in Proposition 2.2, we can show that if $z \notin J(\varphi \circ T)$, we can find, for any $\varepsilon>0, M>0$, a $u \in A$ such that $\|u\|<\varepsilon,\|\varphi(T(z u))\|>M$. Since $y_{n_{p}+1} \in J_{n_{p}}$, let $M_{p}$ denote the bound of the continuous map $x \rightarrow \varphi_{n_{p}}\left(T\left(x y_{n_{p}+1}\right)\right)$. Now choose, by induction, a sequence $\left\{x_{k}\right\} \subseteq A$ satisfying the following conditions.

(1) For each integer $p<k$ we have all three of (a), (b), and (c) 
satisfied

(a) $\left\|x_{k}\right\|\left\|\prod_{j=n_{p}+2}^{n_{k}} y_{j}\right\|<2^{-k}$

(b) $\left\|x_{k}\right\|\left\|\Pi_{j=1}^{n_{k}} y_{j}\right\|<2^{-k}$

(c) $M_{p}\left\|\prod_{j=1}^{n_{p}} y_{j}\right\|\left\|\prod_{j=n_{p^{+2}}}^{n_{k}} y_{j}\right\|\left\|x_{k}\right\|<2^{-k}$.

(2) $\left\|\varphi_{n_{k}}\left(T\left(\left(\prod_{j=1}^{n_{k}} y_{j}\right) x_{k}\right)\right)\right\|>k+1+\sum_{j=1}^{k-1}\left\|T\left(\left(\prod_{i=1}^{n_{j}} y_{i}\right) \bar{x}_{j}\right)\right\|$.

These choices are possible because $\prod_{j=1}^{n_{k}} y_{j} \notin J_{n_{k}}$.

Now let $x=\sum_{k=1}^{\infty}\left(\prod_{j=1}^{n_{k}} y_{j}\right) x_{k} ; x \in A$ by condition (1b) above. Also note that

$$
\begin{aligned}
x & =\sum_{k=1}^{p}\left(\prod_{j=1}^{n_{k}} y_{j}\right) x_{k}+\left(\prod_{j=1}^{n_{p}} y_{j}\right) y_{n_{p}+1}\left[\sum_{k=p+1}^{\infty}\left(\prod_{j=n_{p}+2}^{n_{k}} y_{j}\right) x_{k}\right] \\
& =\sum_{k=1}^{p}\left(\prod_{j=1}^{n_{k}} y_{j}\right) x_{k}+\left(\prod_{j=1}^{n_{p}} y_{j}\right) y_{n_{p}+1} u_{p}
\end{aligned}
$$

where $u_{p}=\sum_{k=p+1}^{\infty}\left(\prod_{j=n_{p}+2}^{n_{k}} y_{j}\right) x_{k}$ exists by condition (1a). We now observe that

$$
\begin{aligned}
\varphi_{n_{p}}(T x)= & \sum_{k=1}^{p-1} \varphi_{n_{p}}\left(T\left(\left(\prod_{j=1}^{n_{k}} y_{j}\right) x_{k}\right)\right)+\varphi_{n_{p}}\left(T\left(\left(\prod_{j=1}^{n_{p}} y_{j}\right) x_{p}\right)\right) \\
& +\varphi_{n_{p}}\left(T\left(\left(\prod_{j=1}^{n_{p}} y_{j}\right) y_{n_{p}+1} u_{p}\right)\right)
\end{aligned}
$$

and so

$$
\begin{aligned}
\left\|\varphi_{n_{p}}(T x)\right\| & \geqq\left\|\rho_{n_{p}}\left(T\left(\left(\prod_{j=1}^{n_{p}} y_{j}\right) x_{p}\right)\right)\right\|-\sum_{k=1}^{p-1}\left\|\rho_{n_{p}}\left(T\left(\prod_{j=1}^{n_{p}} y_{j}\right) x_{k}\right)\right\| \\
& -\left\|\rho_{n_{p}}\left(T\left(\left(\prod_{j=1}^{n_{p}} y_{j}\right) y_{n_{p}+1} u_{p}\right)\right)\right\| \\
& \geqq p+1-\left\|\varphi_{n_{p}}\left(T\left(\left(\prod_{j=1}^{n_{p}} y_{j}\right) y_{n_{p}+1} u_{p}\right)\right)\right\| .
\end{aligned}
$$

But $\left\|\varphi_{n_{p}}\left(T\left(\Pi_{j=1}^{n_{p}} y_{j}\right) y_{n_{p+1}} u_{p}\right)\right\| \leqq M_{p}\left\|\Pi_{j=1}^{n_{p}} y_{j}\right\|\left\|u_{p}\right\|<1$ by condition (1c), and so $\left\|\varphi_{n_{p}}(T x)\right\| \geqq p$. But then $\|T x\| \geqq\left\|\varphi_{n_{p}}(T x)\right\| \geqq p$, a contradiction which completes the proof.

3. Commutative regular semi-simple algebras. The object of this section is to apply the techniques of [1] to obtain more general results, along the lines of [9] and the first section of this paper, for $A$ a commutative regular semi-simple Banach algebra. Throughout this section, we will assume $A$ is such an algebra, and that $T$, whatever its range may be, is separable with respect to algebra multiplication. We shall prove the analogues of the theorems of [1], and merely state the analogues of the propositions of the first section of this paper without proof. We shall let $\Omega$ denote the maximal ideal space of $A$, and regard $A$ as usual via the Gelfand isomorphism as an algebra of continuous function on $\Omega$.

Let $X$ be a normed linear space, $T: A \rightarrow X$. We shall denote by 
$\mathscr{G}$ the collection of all open sets $E \subseteq \Omega$ such that $\operatorname{car}(f) \subseteq E \Longrightarrow f \in$ $J=\{g \in A \mid f \rightarrow T(f g)$ is continuous $\}$. Here car $(f)$ is the closure of $\{w \in Q \mid f(w) \neq 0\}$. We use this definition, rather than the Badé-Curtis definition, as it is more useful for our purposes.

LEMMA 3.1. If $\left\{f_{n}\right\},\left\{g_{n}\right\} \subseteq A$, and $f_{n} g_{m}=0$ for $n \neq m$, then $\exists N$ such that $n \geqq N \Rightarrow f_{n} g_{n} \in J$.

Proof. Once again, a portion of the proof of Proposition 1.2 is sufficiently similar to a proof of this lemma that there is no need to go through the motions.

Lemma 3.2. If $\left\{E_{n}\right\}$ is a sequence of disjoint open sets in $\Omega$, then $\exists N$ such that $n \geqq N \Longrightarrow E_{n} \in \mathscr{G}$.

Proof. Suppose not, and choose a sequence $\left\{n_{k}\right\}$ of integers, $n_{k}<n_{k+1}$, such that no $E_{n_{k}} \in \mathscr{C}$. For each integer $k$, choose $f_{k} \in A$ with $\operatorname{car}\left(f_{k}\right) \subseteq E_{n_{k}}, f_{k} \in J$. Since $A$ is regular, choose a function $g_{k}$ which is 1 on a neighborhood of $\operatorname{car}\left(f_{k}\right)$ and 0 on a neighborhood of $\Omega \sim E_{\imath_{k}}$. By Lemma 3.1, $\exists$ an integer $N$ such that $k \geqq N \Rightarrow f_{k} g_{k} \in J$. By construction, $f_{k}=f_{k} g_{k}$, a contradiction.

LEMMA 3.3. $\mathscr{C}$ is closed under arbitrary unions, and if $E_{0}=$ $\bigcup\{E \mid E \in \mathscr{C}\}$, then $\Omega \sim E_{0}$ is finite.

Proof. This is sufficiently similar to Lemmas 3.3-3.7 of [1] to present no problem.

Proposition 3.1. If $V$ is any neighborhood of $F=\Omega \sim E_{0}$ and $M(V)=\{f \in A \mid f(V)=0\}$, then $T \mid M(V)$ is continuous.

Proof. Use the regularity of $A$ to find a $g \in A$ which is 1 on a neighborhood of $\Omega \sim V$ and 0 on a neighborhood of $F$. Then $\operatorname{car}(g) \cong E_{0}$, and so $g \in J$. Choose $K>0$ such that $f \in A \Rightarrow\|T(f g)\| \leqq K\|f\|$. If $f \in M(V)$, then $f g=f$, and done.

We note that the Badé-Curtis result that $\exists$ a constant $K>0$ such that $f, g \in A, f g=f, \operatorname{car}(f) \cup \operatorname{car}(g) \subseteq E_{0} \Rightarrow\|T(f g)\| \leqq K\|f\|\|g\|$ (from which Proposition 3.1 can also be derived) can be obtained by using their definition of $\mathscr{C}$ and Lemma 1.1. Let $\mathscr{T}(F)$ denote the ideal of all $f \in A$ which vanish on some neighborhood of $F$, the neighborhood varying with $f$, and let $M(F)=\{f \in A \mid f(F)=0\}$. We have shown that $\mathscr{T}(F) \subseteq J$.

We note that, if an ideal $I$ in a Banach algebra contains a bounded 
approximate identity, so does the ideal $\bar{I}$. For suppose $f \in \bar{I}$ and $\varepsilon>0$; let $C$ be the bound of the approximate identity. Choose $g \in I$ with $\|f-g\|<\varepsilon / 2(C+1)$ and $e$ in the approximate identity with $\|e g-g\|<$ $\varepsilon / 2$. Then

$$
\begin{aligned}
\|e f-f\| & \leqq\|e f-e g\|+\|e g-g\|+\|g-f\| \\
& \leqq(\|e\|+1)\|g-f\|+\|e g-g\| \\
& <(C+1)\|g-f\|+\varepsilon / 2<\varepsilon .
\end{aligned}
$$

The reason for using our definition of $\mathscr{G}$ is seen in the following proposition.

Proposition 3.2. Suppose that $T$ is a separable map for which $J$ is closed, and assume that $\mathscr{T}(F)$ contains a bounded approximate identity. Then $T \mid \overline{\mathscr{T}}(F)$ is continuous.

Proof. Since $\mathscr{T}(F) \subseteq J, \overline{\mathscr{T}(F)} \subseteq J$ and $\overline{\mathscr{T}(F)}$ contains a bounded approximate identity. By the Johnson-Varapolous Factorization Theorem cited in Lemma 2.1(c), if $\left\{f_{n}\right\} \subseteq \overline{\mathscr{T}(F)}$ and $f_{n} \rightarrow 0$, we can find $h \in \overline{\mathscr{T}(F)}$ and $\left\{h_{n}\right\} \subseteq \overline{\mathscr{T}(F)}, h_{n} \rightarrow 0$, such that $f_{n}=h h_{n}$. But $h \in J \Rightarrow T\left(h h_{n}\right) \rightarrow 0$, and done.

Proposition 3.3. Let $A$ be such that $\mathscr{T}(F)$ contains a bounded approximate identity and that $\overline{\mathscr{T}(F)}=M(F)$. Then $T$ is continuous on a dense sub-algebra of $A$. If $J$ is closed, then $T$ is continuous.

Proof. The first part of the proposition proceeds similarly to ([1], Theorem 5.1). The second part follows from the fact that $T \mid \overline{\mathscr{T}(F)}$ is continuous by Proposition 3.2 and the fact that $\overline{\mathscr{T}(F)}=M(F)$, which is confinite, by hypotheses.

We note that the algebra of absolutely convergent Fourier series is an example of an algebra satisfying the hypotheses of Proposition 3.3, and also that Katznelson has constructed an example ([1], 607$608)$, which regrettably demonstrates that there exist algebras $A$ such that $\mathscr{T}(F)$ contains a bounded approximate identity, but $\overline{\mathscr{T}}\left(F^{\prime}\right)$ is not cofinite.

Proposition 3.4. Let $X$ be a Banach algebra, $Y$ a normed linear space, $\pi: A \rightarrow X$ a continuous homomorphism, and $T: X \rightarrow Y$ a. separable map. Let $I=\{a \in A \mid x \rightarrow T(\pi(a) x)$ is continuous $\}$. Then $\exists a$ finite subset $F \subseteq \Omega$ such that $\mathscr{T}(F) \subseteq I$. If $J$ is closed, then $\overline{\mathscr{T}(F)} \subseteq I$. Similar results can be obtained when $X$ is an A-module, $T$ is separable with respect to module multiplication, and $I=\{a \in A \mid x \rightarrow$ $T(a x)$ is continuous\}. 
The natural problem now is to try to obtain results for commutative semi-simple algebras which are not a priori regular. Johnson ([8], Lemma 2) has made use of a substitute for regularity in proving that every derivation of a commutative semi-simple Banach algebra into itself is continuous, but his method relies heavily on various properties of derivations, and does not readily apply to this problem.

4. Restrictions on the range. The rest of this paper has dealt with automatic continuity problems when restrictions are placed upon the domain of the map. However, there exist theorems in which restrictions are placed upon the range, such as ([1], Theorem 4.5), which states that every homomorphism of $C(X)$ into an algebra with nil Jacobson radical (some power of every element is 0 ) is continuous.

The problem we wish to consider in this section is the following: Let $\mathscr{A}, \mathscr{B}$ be classes of Banach algebras such that, $A \in \mathscr{A}, B \in \mathscr{B}$, and $T: A \rightarrow B$ is a homomorphism, then $T$ is continuous. Is there a painless way of finding a class $\mathscr{B}^{\prime} \supseteqq \mathscr{B}$ such that $A \in \mathscr{A}, B \in \mathscr{B}^{\prime}$, and $T: A \rightarrow B$ is a homomorphism, then $T$ is continuous? Although we shall concern ourselves with homomorphisms of Banach algebras, it will become apparent from the work in this section that the method used can be applied to more general classes of Banach spaces and maps.

We start by defining the separating ideals associated with a homomorphism.

Definition 4.1. Let $X, Y$ be Banach algebras, $T: X \rightarrow Y$ a homomorphism. Define $S(T, X)=\left\{x \in X \mid \exists\left\{x_{n}\right\} \subseteq X\right.$ such that $x_{n} \rightarrow 0$ and $\left.T x_{n} \rightarrow T x\right\}$, and define $S(T, Y)=\left\{y \in Y \mid \exists\left\{x_{n}\right\} \subseteq X\right.$ such that $x_{n} \rightarrow 0$ and $\left.T x_{n} \rightarrow y\right\}$.

Note that $S(T, X)$ is a closed two-sided ideal in $X$, and that $S(T, Y)$ is a closed subspace of $Y$. If $Y=\overline{T(X)}, S(T, Y)$ is also a two-sided ideal. The Closed Graph Theorem insures that $S(T, Y)=(0) \Rightarrow T$ is continuous. For more properties of these ideals, see [2].

The basic device we shall use to study the problem is the following elementary lemma.

Lemma 4.1. Let $A, B, C$ be Banach algebras, and let $T_{1}: A \rightarrow B$, $T_{2}: B \rightarrow C$ be homomorphisms such that $T_{2} \circ T_{1}: A \rightarrow C$ is continuous. Then $S\left(T_{1}, B\right) \subseteq S\left(T_{2}, B\right)$.

Proof. Let $y \in S\left(T_{1}, B\right)$. Then $\exists\left\{x_{n}\right\} \subseteq A$ with $x_{n} \rightarrow 0, T_{1} x_{n} \rightarrow y$. Since $T_{2} \circ T_{1}$ is continuous, $T_{2} \circ T_{1}\left(x_{n}\right) \rightarrow 0$. So $y-T_{1} x_{n} \rightarrow 0$ and $T_{2}\left(y-T_{1} x_{n}\right)=T_{2} y-T_{2} \cdot T_{1}\left(x_{n}\right) \rightarrow T_{2} y$, which implies that $y \in S\left(T_{2}, B\right)$. 
When $T: A \rightarrow B$ is continuous, it is easy to see that $S(T, A)=$ $\operatorname{ker}(T)$, the kernel of $T$. An immediate consequence of this remark and the preceding lemma is that homomorphisms into commutative semi-simple algebras are continuous. The preceding lemma also motivates the following definitions.

Definition 4.2. Let $\mathscr{A}, \mathscr{B}_{0}$ be classes of Banach algebras such that $A \in \mathscr{A}, B \in \mathscr{B}$, and $T: A \rightarrow B$ a homomorphism $\Rightarrow T$ is continuous. If $X$ is a Banach algebra and $B \in \mathscr{B}_{0}$, define

$$
\mathscr{S}_{0}(X, B)=\bigcap\{S(T, X) \mid T: X \rightarrow B \text { a homomorphism }\} .
$$

Let $\mathscr{S}_{0}(X)=\bigcap\left\{\mathscr{S}_{0}(X, B) \mid B \in \mathscr{B}_{0}\right\}$, and let $\mathscr{\mathscr { B }}_{1}$ be the collection of all Banach algebras $X$ for which $\mathscr{S}_{0}(X)=(0)$.

Note that $\mathscr{S}_{0}(X, B)$ and $\mathscr{S}_{0}(X)$ are closed two-sided ideals. They were defined with a view towards the following proposition.

Proposition 4.1. If $A \in \mathscr{A}, B \in \mathscr{B}_{1}$, and $T: A \rightarrow B$ is a homomorphism, then $T$ is continuous.

Proof. We have observed that it suffices to show $S(T, B)=(0)$. If $K \in \mathscr{B}_{0}$ and $\Phi: B \rightarrow K$ is a homomorphism, by Lemma 4.1 we see that $S(T, B) \subseteq S(\Phi, B)$. So $S(T, B) \subseteq \mathscr{S}_{0}(B)=(0)$.

Clearly $\mathscr{B}_{0} \subseteq \mathscr{B}_{1}$, for if $B \in \mathscr{B}_{0}$, let $i: B \rightarrow B$ be the identity. It clearly has zero kernel, which is the domain separating ideal since $i$ is continuous, and so $\mathscr{S}_{0}(B)=(0)$.

This procedure can evidently be continued.

Definition 4.3. Assume now that $\mathscr{P}_{n}$ has been defined inductively in such a way that $A \in \mathscr{A}, B \in \mathscr{B}_{n}$, and $T: A \rightarrow B$ a homomorphism $\Rightarrow T$ is continuous. If $X$ is a Banach algebra and $B \in \mathscr{B}_{n}$, define $\mathscr{S}_{n}(X, B)=\bigcap\{S(T, X) \mid T: X \rightarrow B$ a homomorphism $\}$, and let $\mathscr{S}_{n}(X)=\bigcap\left\{\mathscr{S}_{n}(X, B) \mid B \in \mathscr{B}_{n}\right\}$. Let $\mathscr{B}_{n+1}$ denote the class of all Banach algebras $X$ for which $\mathscr{S}_{n}(X)=(0)$.

The following proposition has essentially been proved already.

Proposition 4.2. (a) If $A \in \mathscr{A}, B \in \mathscr{B}_{n+1}$, and $T: A \rightarrow B$ is a homomorphism, then $T$ is continuous.

(b) $\mathscr{B}_{n} \subseteq \mathscr{B}_{n+1}$

(c) $\mathscr{S}_{n+1}(X) \subseteq \mathscr{S}_{n}(X)$.

Although we could continue this procedure transfinitely, which would lead to questions of the existence of an ordinal $\lambda$ such that $\gamma \geqq \lambda \Rightarrow \mathscr{B}_{r}=\mathscr{B}_{\lambda}$, let us examine the results after a countable number 
of steps. Let $\mathscr{S}(X)=\bigcap_{n=0}^{\infty} \mathscr{S}_{n}(X)$, and let $\mathscr{B}=\{X \mid \mathscr{S}(X)=(0)\}$.

Proposition 4.3. Let $A \in \mathscr{A}, B \in \mathscr{B}$, and $T: A \rightarrow B$ be a homomorphism. Then $T$ is continuous.

Proof. Let $K \in \mathscr{B}_{n}$ for some integer $n$, and let $\Phi: B \rightarrow K$ be a homomorphism. By Proposition 4.2(a) and Lemma 4.1, $S(T, B) \subseteq$ $S(\Phi, B)$; consequently $S(T, B) \subseteq \mathscr{S}_{n}(B)$ for each integer $n$, and so $S(T, B) \subseteq \bigcap_{n=0}^{\infty} \mathscr{S}_{n}(B)=(0)$. The result follows.

It should be noted that the closed ideals $\left\{\mathscr{S}_{n}(X)\right\}$ are defined in a way that makes them apparently have properties in common with a radical. Two interesting properties of a radical (an ideal $R(X)$ defined for each algebra $X$ in a given class of algebras) are

(1) $X=R(X) \Rightarrow A=R(A)$ for any homomorphic image $A$ of $X$

(2) $R(X / R(X))=(0)$ for all $X$.

For a further description of radicals, see [3].

Clearly, property (2) above would be extremely useful, as it provides a method of obtaining algebras into which every homomorphism whose domain is an algebra in $\mathscr{A}$ is continuous.

Let us just note that, if $n \geqq 1$, the class $\mathscr{B}_{n}$ is closed under sub-algebras, i.e., if $B \in \mathscr{\mathscr { S }}_{n}$ and $K$ is a Banach sub-algebra of $B$, then $K \in \mathscr{B}_{n}$. Let $i: K \rightarrow B$ denote the isometric injection, and let $C \in \mathscr{B}_{n-1}$. Let $T: B \rightarrow C$ be a homomorphism; then if $x \in S(T \circ i, K)$, we can find $x_{n} \rightarrow 0$ in $K, T \circ i\left(x_{n}\right) \rightarrow T \circ i(x)$. But then $i\left(x_{n}\right) \rightarrow 0$ in $B, T\left(i\left(x_{n}\right)\right) \rightarrow$ $T(i(x)) \Rightarrow i(x)=x \in S(T, B)$; hence $S(T \circ i, K) \subseteq S(T, B)$; so if $\mathscr{S}_{n}(B)=$ $(0)$, clearly $\mathscr{S}_{n}(K)=(0)$. We can similarly assume $\mathscr{B}_{0}$ is closed under sub-algebras.

Property (1) is rendered uninteresting by ([2], Proposition 4.7), and besides it is property (2) that is useful for our problem. Under certain conditions which are not too difficult to satisfy, property (2) holds.

Proposition 4.4. Suppose that the class $\mathscr{B}_{n}$ is closed under quotients, i.e., if $B \in \mathscr{B}_{n}$ and $I$ is a closed two-sided ideal in $B$, $B / I \in \mathscr{B}_{n}$. Then $\mathscr{S}_{n}(X)$ is the intersection of the kernels of continuous homomorphisms from $X$ into algebras in $\mathscr{B}_{n}$, and $\mathscr{S}_{n}\left(X / \mathscr{S}_{n}(X)\right)=(0)$.

Proof. Let $X$ be a Banach algebra, $B \in \mathscr{B}_{n}$, and let $T: X \rightarrow B$ be a homomorphism. By the observation that $\mathscr{B}_{n}$ is closed under subalgebras, we can assume that $\overline{T(X)}=B$, so $S(T, B)$ is a closed twosided ideal in $B$. By ([2], Theorem 4.6), the homomorphism $\widetilde{T}: X / S(T, X) \rightarrow B / S(T, B)$ defined by $\widetilde{T}(x+S(T, X))=T x+S(T, B)$ is a continuous isomorphism. Let $\pi: X \rightarrow X / S(T, X)$ denote the quo- 
tient map: $\|\pi\| \leqq 1$, and since $\hat{B}=B / S(T, B) \in \mathscr{B}_{n}$ by assumption, let $\widehat{T}: X \rightarrow \hat{B}$ be defined by $\hat{T}=\widetilde{T} \circ \pi . \quad \hat{T}$ is clearly continuous; since $\hat{T}$ is an isomorphism, $\operatorname{ker}(\hat{T})=\operatorname{ker}(\pi)=S(T, X)$.

Now let $B \in \mathscr{B}_{n}$, and let $T: X \rightarrow B$ be a homomorphism. Clearly $\mathscr{S}_{n}(X) \cong S(T, X)$, so let $i: X / \mathscr{S}_{n}(X) \rightarrow X / S(T, X)$ be the natural map defined by $i\left(x+\mathscr{S}_{n}(X)\right)=x+S(T, X)$. Define $\widetilde{T}$ as in the preceding paragraph, then $\widetilde{T} \circ i: X / \mathscr{S}_{n}(X) \rightarrow B / S(T, B)$ is continuous and $S\left(X / \mathscr{S}_{n}(X), \widetilde{T} \circ i\right)=\operatorname{ker}(\widetilde{T} \circ i)=\operatorname{ker}(i)=\left\{x+\mathscr{S}_{n}(X) \mid x \in S(T, X)\right\}$. So $\mathscr{S}_{n}\left(X / \mathscr{S}_{n}(X)\right) \subseteq \operatorname{ker}(i)$ for each $i: X / \mathscr{S}_{n}(X) \rightarrow X / S(T, X)$; but since $\mathscr{S}_{n}(X)$ is the intersection of all such $S(T, X)$, we see that $\mathscr{S}_{n}\left(X / \mathscr{S}_{n}(X)\right)=(0)$.

Consider the case where $\mathscr{A}$ is the collection of all algebras $C(X)$ for some compact Hausdorff $X$, and $\mathscr{B} 0$ is the collection of all commutative Banach algebras with nil Jacobson radical. In what appears to be some sort of confirmation of the saying that "all roads lead to Rome", we find ourselves faced with the following problem, which also confronted us in $\S 2$ : for which algebras $A$ do there exist homomorphisms into algebras with nil radical taking elements of the radical of $A$ into nonzero elements? The author asked Professor Divinsky this question, and received a reply that, if the intersection of all powers of the radical was zero, for each nonzero $x \in X$ we could find such a homomorphism. However, if this intersection is nonzero, the problem is still unsolved.

\section{REFERENCES}

1. W. G. Badé and P. C. Curtis, Jr., Homomorphisms of commutative Banach algebras, Amer. J. Math., 82 (1960), 589-608.

2. S. B. Cleveland, Homomorphisms of noncommutative *algebras, Pacific J. Math., 13 (1963), 1097-1109.

3. N. Divinsky, Rings and Radicals, London, 1965.

4. E. L. Griffin, Everywhere defined linear transformations affiliated with rings of operators, Pacific J. Math., 18 (1966), 489-493.

5. B. E. Johnson, Continuity of centralizers on Banach algebras, J. London Math. Soc., 41 (1966), 639-640.

6. - Continuity of homomorphisms of algebras of operators, London J. Math., 24 (1967), 537-541.

7. - Continuity of homomorphisms of algebras of operators (II), J. London Math. Soc., 2 (1969), 81-84.

8. — Continuity of derivations on commutative algebras, Amer. J. Math., 91 (1969), 1-10.

9. K. B. Laursen and J. D. Stein, Jr., Automatic continuity in Banach spaces and algebras, to appear in Amer. J. Math.

10. J. R. Ringrose, Continuity of derivations on $C^{*}$-algebras, to appear.

11. J. D. Stein, Jr., Continuity of homomorphisms of Von Neumann algebras, Amer. J. Math., 91 (1969), 153-159.

12. - Homomorphisms of $B^{*}$-algebras, Pacific J. Math., 28 (1969), 431-439.

13. - Several theorems on boundedness and equicontinuity, Proc. Amer. Math. Soc., 26 (1970), 415-419. 
14. D. C, Taylor, A characterization of Banach algebras with approximate unit, Bull. Amer. Math. Soc., 74 (1968), 761-766.

Received September 28, 1972. This work was supported in part by NSF Grant No. GP-29011.

University of California, Los ANgeles 


\section{PACIFIC JOURNAL OF MATHEMATICS}

\section{EDITORS}

RICHARD ARENS (Managing Editor) University of California

Los Angeles, California 90024

R. A. BeaUmont

University of Washington Seattle, Washington 98105
J. DUGUNDJI*

Department of Mathematics University of Southern California Los Angeles, California 90007

D. Gilbarg and J. Milgram Stanford University

Stanford, California 94305

\section{ASSOCIATE EDITORS}

E. F. BECKENBACH

B. H. NeUmanN

F. WOLF

K. YoSHIDA

\section{SUPPORTING INSTITUTIONS}

\section{UNIVERSITY OF BRITISH COLUMBIA CALIFORNIA INSTITUTE OF TECHNOLOGY UNIVERSITY OF CA.LIFORNIA MONTANA STATE UNIVERSITY UNIVERSITY OF NEVADA NEW MEXICO STATE UNIVERSITY OREGON STATE UNIVERSITY UNIVERSITY OF OREGON OSAKA UNIVERSITY}

UNIVERSITY OF SOUTHERN CALIFORNIA STANFORD UNIVERSITY UNIVERSITY OF TOKYO UNIVERSITY OF UTAH WASHINGTON STATE UNIVERSITY UNIVERSITY OF WASHINGTON AMERICAN MATHEMATICAL SOCIETY NAVAL WEAPONS CENTER

* C. R. DePrima California Institute of Technology, Pasadena, CA 91109, will replace J. Dugundji until August 1974. 


\section{Pacific Journal of Mathematics}

\section{Vol. 50, No. $1 \quad$ September, 1974}

Gail Atneosen, Sierpinski curves in finite 2-complexes.............. 1

Bruce Alan Barnes, Representations of $B^{*}$-algebras on Banach spaces .... 7

George Benke, On the hypergroup structure of central $\Lambda(p)$ sets ....... 19

Carlos R. Borges, Absolute extensor spaces: a correction and an

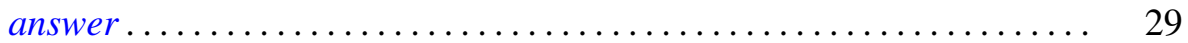

Tim G. Brook, Local limits and tripleability .................. 31

Philip Throop Church and James Timourian, Real analytic open maps .... 37

Timothy V. Fossum, The center of a simple algebra ............... 43

Richard Freiman, Homeomorphisms of long circles without periodic

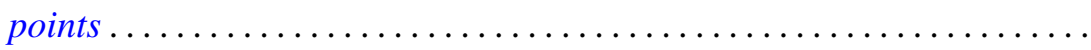

B. E. Fullbright, Intersectional properties of certain families of compact

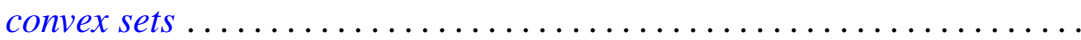

Harvey Charles Greenwald, Lipschitz spaces on the surface of the unit

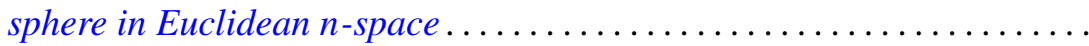

Herbert Paul Halpern, Open projections and Borel structures for

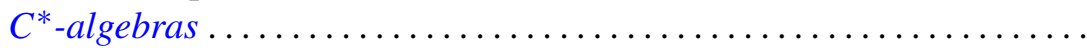

Frederic Timothy Howard, The numer of multinomial coefficients divisible

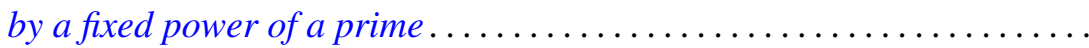

Lawrence Stanislaus Husch, Jr. and Ping-Fun Lam, Homeomorphisms of manifolds with zero-dimensional sets of nonwandering points........ 109

Joseph Edmund Kist, Two characterizations of commutative Baer rings ...

Lynn McLinden, An extension of Fenchel's duality theorem to saddle functions and dual minimax problems ................

Leo Sario and Cecilia Wang, Counterexamples in the biharmonic classification of Riemannian 2-manifolds...

Saharon Shelah, The Hanf number of omitting complete types ...

Richard Staum, The algebra of bounded continuous functions into a

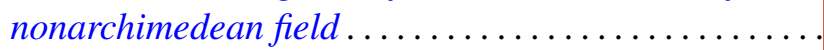

James DeWitt Stein, Some aspects of automatic continuity ..

Tommy Kay Teague, On the Engel margin

John Griggs Thompson, Nonsolvable finite groups all of whose local subgroups are solvable, $V \ldots \ldots \ldots \ldots \ldots \ldots \ldots \ldots$

Kung-Wei Yang, Isomorphisms of group extensions 